\title{
interview
}

\section{Generating light bullets}

\author{
Generating 3D light packets that propagate without dispersing in time or space is not an easy task. \\ Andy Chong from Cornell University told Nature Photonics how he and his co-workers came up with a \\ simple and versatile approach to this problem.
}

Tell us about your work.

Owing to the nature of waves themselves, any localized wavepacket tends to broaden in space and time under the joint effect of dispersion and diffraction. The aim of our work, therefore, is to generate localized $3 \mathrm{D}$ packets of light that do not disperse and diffract. The idea of generating 3D wavepackets that avoid dispersion and diffraction was first proposed in the context of nonlinear optics, where such fields are called space-time solitons or 'light bullets', owing to their particle-like nature. Despite much research, 3D optical solitons have not yet been produced; they tend to disintegrate during propagation because of the inherent instabilities of the ultrafast nonlinearity needed to keep their shape. Also, it's difficult to balance two linear effects using a single nonlinear process, except when the effects of diffraction and dispersion are equal. Moreover, nonlinear approaches generally require very high peak powers, which may damage the medium in which the waves propagate. There are certain approaches that operate in the linear regime, but the usefulness of these schemes has been limited by the need to tailor the wavepackets precisely to match the material properties of the propagation medium. In contrast, we have now generated 3D localized waves in the linear regime in a versatile way that allows us to avoid dependence on the material properties. The most important aspect for realizing the scheme was to decouple space and time so that it is no longer necessary to match the spatial and temporal parameters of the wavepacket. Separation of this 3D spatiotemporal problem into its elemental constituents requires at least one $1 \mathrm{D}$ dispersion- or diffraction-free wavepacket. In our case, the $1 \mathrm{D}$ dispersion-free Airy pulse is used. We essentially combine 2D diffractionfree Bessel beams in the spatial domain with $1 \mathrm{D}$ dispersion-free Airy pulses in the temporal domain.

How do you generate the light bullets? The idea of generating linear light bullets based on this approach was proposed by Demetrios Christodoulides - the pioneer of Airy beams. The theory behind the idea

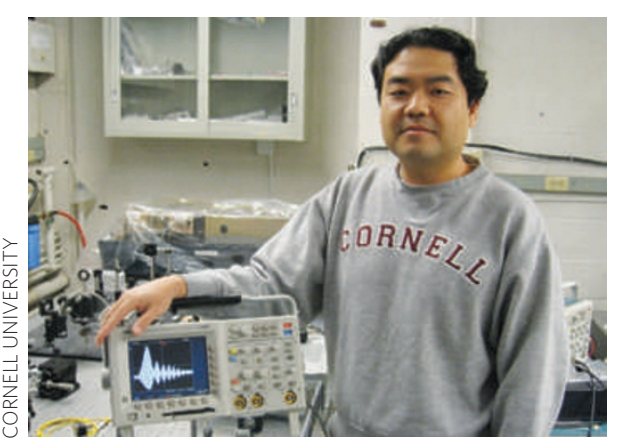

Andy Chong with the experimental set-up used to create non-diffracting and non-dispersing Airy-Bessel linear light bullets.

has been reported before, and now we report experimental realization of such wavepackets and show how they propagate through various media. We initially convert a Gaussian-Gaussian wavepacket into an Airy-Gaussian wavepacket using a gratingtelescope compensator to adjust the cubic phase needed to generate an Airy temporal profile. We then convert the wavepacket into the Airy-Bessel wavepacket using a beam-expansion telescope and an axicon lens. We successfully demonstrate the temporal self-healing and free acceleration features of the Airy pulse. We also demonstrate the non-spreading nature of the Airy-Bessel wavepacket by generating a fluorescence channel in a dispersive and fluorescent medium.

\section{What are the advantages of your approach?}

The advantages are that the wavepacket evolution does not depend on the material in which the beams propagate, and that there is no need to precisely tailor the dispersion and diffraction lengths during the generation. Furthermore, the wavepackets can be created in many different materials, in contrast with $3 \mathrm{D}$ solitons. Unlike other types of self-localized waves, these bullets exist without the need for dispersiondiffraction equalization, and can exist even under normal or anomalous dispersion. Generation of versatile light bullets may be possible using other families of localized solutions; for example by combining an Airy beam with another 2D non-diffracting beam such as a higher-order Bessel or Mathieu beam, or by combining an Airy beam with a 2D Airy beam to form an Airy-Airy-Airy wavepacket configuration. Although these schemes may offer useful features, they generally require complicated set-ups.

\section{What are the implications of your findings?}

Our findings represent the first observation of a class of versatile 3D linear light bullets. This may lead to a myriad of applications involving the use of tightly focused short pulses in linear media, such as microscopy and probing techniques in materials science. For instance, a recently developed optical projection tomography passes a beam through a specimen that causes scattering to obtain a $3 \mathrm{D}$ image. It was pointed out that a higher resolution can be obtained if the beam is close to a tightly focused line in the specimen. A non-dispersing and non-diffracting Airy-Bessel wavepacket may offer versatile optical projection tomography equipment with high resolution for any specimen of unknown dispersion. In plasma physics, plasma channel generation in a dispersive medium can be achieved with better performance using Airy-Bessel beams because the peak power of the beams can be maintained throughout the channel.

\section{What are the challenges and possibilities for future work?} At the moment, only $\sim 10 \%$ of the energy of the original Gaussian-Gaussian wavepacket is in the main spatiotemporal lobe, which may be a limitation for certain applications. We are now looking at applying Airy-Bessel beams to microscopy through collaborations with other groups. We also wish to generate Airy-Airy-Airy wavepackets, which propagate in an interesting 3D curved trajectory. The major goal of group is to generate $3 \mathrm{D}$ nonlinear light bullets - one of the holy grails of the field of nonlinear optics.

\section{INTERVIEW BY RACHEL WON}

Andy Chong and his co-workers have a Letter on the generation of linear light bullets on page 103 of this issue. 